\title{
The volumetric evaluation of sinüs floor augmentation using platelet rich-fibrin and allogenic bone graft mixture with simultaneous dental implant placement in severely atrophic posterior maxilla
}

\author{
Omur Dereci $^{1}$, Nesrin Saruhan ${ }^{1}$, and Gorkem Tekin ${ }^{2}$ \\ ${ }^{1}$ Eskisehir Osmangazi University \\ ${ }^{2}$ Affiliation not available
}

January 11, 2021

\begin{abstract}
Objectives: To evaluate the efficacy of the combination of platelet-rich fibrin (PRF) and mineralized bone allograft on the volume of the new bone formation in the sinus floor augmentation of the severely atrophic residual alveolar crest with simultaneous dental implant placement. Materials and Methods: All clinical and radiological data of patients treated with sinus floor augmentation using the combination of PRF and demineralized bone allograft with simultaneous dental implant placement between 01/04/2013 and 01/03/2020 were retrieved from the archives. The main inclusion criterion for the study was a residual ridge height less than $2 \mathrm{~mm}$. The volume of the maxillary sinus cavity was measured by a software program on pre-operative and post-operative cone beam computerized tomography (CBCT) sections. The graft volume was calculated by subtracting pre-operative and post-operative total sinus volume. Residual and post-operative ridge heights were measured by the software program on the mid-coronal and mid-sagittal sections of pre-operative and post-operative CBCT images. Results: Eighteen patients with 26 sinus augmentations were included in the study. Forty-six dental implants were placed simultaneously with sinus floor augmentation. The follow-up periods of cases varied between 19 and 29 months. All cases showed new bone regeneration on axial, coronal and sagittal sections of post-operative CBCT data. The mean volume of newly regenerated bone was calculated as $2.1( \pm 0.9) \mathrm{ml}$. Post-operative ridge heights was significantly increased compared to pre-operative residual ridge heights $(\mathrm{p}<0.05)$. There was no sign of a relationship between residual and post-operative ridge heights $(\mathrm{p}>0.05)$. Conclusion: The PRF and demineralized bone allograft combination used in sinus floor augmentation may provide healthy new bone that supports simultaneously placed dental implants in function in the severely atrophic alveolar crests with a residual ridge height less than $2 \mathrm{~mm}$. Keywords: Sinüs floor augmentation ; allograft ; cone-beam computed tomography ; dental implant
\end{abstract}

\section{Introduction}

Sinus floor augmentation is a bone regeneration technique performed by creating a void between the Schneiderian membrane and native bone and filling this empty cavity with block or particulate bone grafts of various origin. Different types of bone grafts such as autogenous, alloplastic, allogenic, or xenografts have been used in the sinus augmentation procedures in varying success levels. Autogenous bone graft is the harvested native bone that is to be transferred to the surgical site with a bone insufficiency and reported as the gold standard in sinus floor augmentation procedure due to its osteoinductive and osteogenetic properties.(1, 2) However, the donor site morbidity and increase in surgical sites are the most important handicaps in autogenous bone harvesting. The use of allogenic bone grafts does not cause donor site morbidity and they are reported to have osteoconductive and osteoinductive properties. $(3,4)$ However, it was suggested that the new bone produced by bone allografts was insufficient in quality and quantity. $(5,6)$ The use of the 
combination of bone allografts with xenogenic or alloplastic materials is the preferred technique and shows satisfying results in acquiring new bone formation in the sinus floor augmentation. $(7,8)$

The timing of dental implant placement in the augmented sinus is controversial. Simultaneous implant placement with sinus floor augmentation is generally recommended if the residual crestal height is equal to or greater than $4 \mathrm{~mm}$.(9-11) A two-staged procedure of dental implant placement is suggested as appropriate due to the difficulty of obtaining the primer stability and the need of osseous regeneration of bone graft when the residual ridge height is less than $4 \mathrm{~mm}$.(12) However, several studies reported that simultaneous dental implant placement can be successfully performed in patients with residual ridge heights between 1 and 3 mm. $(9,11)$

The combination of biological mediators with non-autogenous bone grafts has been proposed in order to increase the osteogenic and osteoinductive capacity of the bone graft.(13) Platelet-rich fibrin (PRF) is a blood substitute and includes several growth factors and cytokines such as transforming growth factor beta1 (TGFB-1), vascular endothelial growth factor (VEGF), platelet-derived growth factor (PDGF), and bone morphogenic protein-1 (BMP-1) that act as biological mediators when it is implemented into a wound.(14, 15) PRF also forms a solid fibrin network that enables cell migration and proliferation.(16) Several reports showed the efficacy of PRF on new bone regeneration when combined with demineralized bovine bone matrix (DBBM) or allogenic bone graft in sinus floor augmentation. $(17,18)$

The aim of this study is to evaluate the efficacy of the combination of PRF and demineralized bone allograft on the volume of the new bone formation in the sinus floor augmentation of the severely atrophic residual alveolar crest with simultaneous dental implant placement.

\section{Materials and Methods}

\section{Patients}

The study was approved by the local Clinical Research Ethics Committee with approval number 2020-469-12 and performed in accordance with the ethical standards laid down in the 1964 Declaration of Helsinki and its later amendments. All clinical and radiological data of patients with posterior alveolar ridge height lower than $2 \mathrm{~mm}$ treated with sinus floor augmentation with simultaneous dental implant placement and posterior maxillary implant-supported fixed dentures between $01 / 04 / 2013$ and $01 / 08 / 2020$ were retrieved from the archives. The inclusion criteria of the study were;

Availability of intact clinical data

Availability of unharmed digitalized volumetric computerized tomography data in DICOM format taken at pre-operative and post-operative control evaluations of patients treated with sinus floor augmentation using the mixture of demineralized cortico-cancellous bone allograft and PRF.

Exclusion criteria were;

1- Patients with chronic metabolic diseases such as diabetes and hypertension

2- Smokers and patients with poor oral hygiene

\section{PRF preparation}

Venous access was established with a vascular cannula and blood was collected with a $20 \mathrm{ml}$ syringe before the initiation of the surgery. The blood was transferred immediately in dry glass tubes without anticoagulant and immediately centrifuged at a speed of $2700 \mathrm{rpm}$ for 10 minutes. The whitish-yellowish PRF clot was separated from the basal red cell accumulation layer with the aid of a tissue scissor and cut into small fragments which were mixed with $2 \mathrm{ml}$ demineralized cortico-cancellous bone allograft (Maxxeus, Community Tissue Services, Ohio, USA) (Figure 1). Two PRF clots were used for each sinüs augmentation procedure. One of them was used for the bone allograft and PRF mixture. The other PRF clot was used as a membrane on the lateral sinüs osteotomy site. 


\section{Sinus augmentation and implant placement}

Sinus floor augmentation surgery was performed by the same researcher (NS) with lateral osteotomy technique. A rectangular osteotomy was prepared at the lateral sinus wall after full-thickness mucosal flap reflection under local anesthesia (Fullcain Fort 2 ml, Onfarma Pharmaceutical Ltd. Company, Samsun, Turkey) with a piezosurgery device (Piezosurgery® Touch Unit, Mectron, Carasco, Italy) under constant irrigation. The rectangular bony window was gently removed to expose the osteotomy site and the Schneiderian membrane was elevated with sinus elevators until the membrane was completely freed from the sinus floor. The mixture of demineralized cortico-cancellous bone allograft and PRF was inserted into the prepared cavity. OsseoSpeed $^{\mathrm{TM}}$ TX dental implants with TiO2-blasted fluoride-modified surface (OSTX) were placed simultaneously under constant physiological saline irrigation. The bony window was replaced on the osteotomy site and covered with a PRF membrane. Flaps were sutured with 3-0 vycril sutures. Patients were administered antibiotics (1000 mg amoxicillin-clavulanate, 2x1), non-steroid anti-inflammatory drugs (550 mg naproxen sodium, $3 \mathrm{x} 1)$, and oral antiseptics (\%0.12 Chlorhexidine digluconate, $3 \times 1)$ for post-operative prophylaxis. Sutures were removed at post-operative 10th day.

Healing caps were mounted under local anesthesia with full-thickness flap elevation under local anesthesia (Fullcain Fort 2 ml, Onfarma Pharmaceutical Ltd. Company, Samsun, Turkey) 6 months after surgery, and fixed partial prosthesis were fabricated 1 week after healing cap placement by one independent clinician.

\section{Volume and Ridge Height Measurement}

Cone-beam computerized tomography $(\mathrm{CBCT})$ images were procured in a standing position with a CBCT machine (Planmeca Promax 3D mid, Helsinki, Finland) and taken under the following exposure parameters: $94 \mathrm{kVp}$ tube voltage, $14 \mathrm{~mA}$ tube current, and 27 second time. The CBCT data was transferred to a software program (SYNAPSE version 4.4.000, Fujifilm, Tokyo, Japan) in DICOM format. The tomographic data was evaluated with $0.4 \mathrm{~mm}$ slice thickness and $2 \mathrm{~mm}$ slide sections. As the first stage of the segmentation process, the borders of the sinus cavity were traced (Figure 2) with a border marker tool and the volume of the sinus cavity was determined on the axial section. After that, the deficient and distorted areas were modified and corrected with the same border marker tool on sagittal (Figure 3), and coronal (Figure 4) sections. The volume of the marked air-filled cavity was highlighted automatically with the reconstruction feature of the software program when the segmentation procedure was completed (Figure 5). A subtraction method was used to measure the total graft volume. In this method, graft volume was determined by subtracting the volume of the complete sinus cavity which was measured by the computer program on pre-operative and post-operative follow-up CBCT data. The same tracing software was used to measure the ridge heights on the posterior maxilla. The residual ridge heights were measured with a measurement marker tool on the mid-sagittal and mid-coronal sections in the central region of sinus floor augmentation (Figure 6-A, B). Post-operative ridge heights were measured with the marker tool adjacent to the dental implant on sagittal and coronal sections (Figure 6-C, D).

The measurements were performed by only one researcher (GT). For intraobserver agreement, the examiner performed tomographic measurements in 10 patients with a sinus floor augmentation which were not included in the current study before the initiation of actual measurements of the study.

\section{Statistical Analysis}

An independent statistician reviewed the methodology and results of the study. SPSS version 21.0 Statistical Software (IBM, Chicago, USA) was used for statistical analysis of the results. Kolmogorov-Smirnov and Shapiro-Wilk tests $(\mathrm{p}>0.05)$ showed that the measurement scores were normally distributed. The difference in the alveolar ridge heights between pre-operative and post-operative control evaluations was analyzed with paired t-test. Pearson correlation coefficient was used in the analysis of the relationship between residual and post-operative ridge heights.

\section{Results}

Eighteen patients with 26 sinus floor augmentations were included in the study. The mean age was 58,1 18,7 . 
Twelve patients were male and 6 of them were female. The follow-up periods varied between 19 and 29 months.

There was a perforation in the sinus membrane in 1 case during the membrane elevation. In this case, the perforation was gently repaired with the use of a PRF membrane. There were no continuing complications as the result of the perforation in this case at the post-operative follow-up period. No other complications were observed during the treatment period. All implants were also functional without any sign of peri-implantitis at the time of control evaluations.

Forty-six dental implants were placed simultaneously with sinus floor augmentation. The region of dental implant placement, lengths, and widths of implants used were shown in Table 1.

Pre-operative and post-operative mean volumes of operated sinus cavities were $19.5( \pm 6)$ and $17.3( \pm 5.6) \mathrm{ml}$, respectively (Table 2$)$. The mean volume of newly regenerated bone was calculated as $2.1( \pm 0.9) \mathrm{ml}$ (Table 2 ). The mean values of residual and post-operative ridge heights were shown in Figure 7. Post-operative ridge heights showed a significant increase compared to residual ridge heights $(\mathrm{p}<0.05)$ (Table 3$)$. There was no sign of a relationship between residual and post-operative ridge heights $(\mathrm{p}>0.05)$ (Table 4$)$.

\section{Discussion}

Residual alveolar ridge height less than $3 \mathrm{~mm}$ is suitable for two-staged dental implant surgery to prevent complications after the sinus augmentation process and achieve success in the long term.(19) However, sinus floor augmentation with simultaneous implant placement in severely atrophic residual crests with a residual ridge height under 3 or $4 \mathrm{~mm}$ can be successful if the native bone is of high quality. In the study of Peleg et al.(11), twenty patients with posterior alveolar ridge height of $1-2 \mathrm{~mm}$ were treated with sinus floor augmentation and simultaneous implant placement using a mixture of autogenous bone and demineralized freezed-dried bone allograft (DFDBA) and it was concluded that inserted dental implants were in good condition without any marginal bone loss with satisfactory bone consolidation around them after 26.5 months average follow-up period. Mardinger et al.(20) reported that dental implants placed simultaneously with sinus floor augmentation in the residual alveolar bone with 1-3 $\mathrm{mm}$ vertical height showed $92 \%$ success rate after a mean follow-up of 36.8 months. Tilaverdis et al.(9) reported that mineralized cancellous bone allograft could be successfully used in the sinus floor augmentation with simultaneous dental implant placement in severely atrophic posterior ridges with alveolar bone heights between 1 and $3 \mathrm{~mm}$. In the current study, a prominent volume of bone regeneration around simultaneously placed dental implants in sinus floor augmentation in the severely atrophic posterior maxilla with a residual ridge height less than $2 \mathrm{~mm}$. There was also a marked increase in the post-operative ridge height in the control evaluation. However, there was no correlation between residual ridge height and post-operative ridge height. This finding may show that the PRF and demineralized bone allograft combination produced high levels of new bone regardless of residual alveolar bone quantity.

Simultaneous implant placement with sinus augmentation in cases with residual ridge heights less than 3-4 $\mathrm{mm}$ is unfavorable due to the main disadvantage of decreased implant support and difficulty in achieving primer stability which is a prerequisite for healthy osseointegration. Dental implants placed in severely atrophic and fragile alveolar crests may undergo positional changes during the placement process with the screwing handpiece or after the surgery with traumatic forces. It was reported that the maneuver of unscrewing the cover screws also may mobilize the dental implant and affect angulation.(20) In the current study, the osteotomy was prepared and finished in lower diameter than the diameter of the last drill that was designated as the final drill for that diameter of the dental implant by the manufacturer in order to provide a solid feeling of pressure when inserting the implant and increase primary stabilization. With this technique, the control of the angulation of the dental implant was established during the placement of the cover screw. It was also reported that meticulous graft placement into the sinus cavity supports dental implants that are simultaneously placed with sinus augmentation and contributes to primary stabilization (9) which is one of the primer necessities in the successful reconstruction in the severely atrophic maxilla.

The use of panoramic radiography in the new bone formation after sinus floor augmentation is not recom- 
mended due to the limitations of inadequate display of the graft area due to the two-dimensional nature of the radiography and difficulty in establishment of outlines of the matured graft due to the superpositions and artifacts on the radiography.(21) Volumetric measurement of the bone regeneration in the sinus floor augmentation is relatively new and aids the clinicians to properly determine the success in the post-operative period. Various computer software and methods have been used for the measurement of bone regeneration in the sinus cavity.(22-26) The segmentation method is an indirect way of measuring graft maturation. In this method, the outlining of the air-filled sinus cavity is marked with a measurement tool on the axial, sagittal and coronal tomographic sections and the volume of the sinus cavity is automatically calculated by the software program. The graft volume is determined indirectly by subtracting pre-operative and postoperative sinus volumes. Several studies reported direct measurement of the sinus bone graft by manually reconstructing the grafted area based on threshold values selected according to the gray values of adjacent structures such as native bone or soft tissue. $(27,28)$ The matured bone graft may usually be irregular and not well-defined. In tomographic sections, the measurement and distinguishing of air-filled areas may be easier when compared with dense bone-like regions because Hounsfeld unit (HU) value of air-filled cavities is always below zero.(29) However, the radiopacity of the maturing graft may vary and it can be difficult to manually delineate the borders of the bone graft.

The combination of a bone grafting material with PRF is an emerging technique in sinus augmentation surgery. The addition of PRF fragments into the bone grafts not only provides a binding medium for the bone graft particles but also facilitates bacterial defense mechanisms and prevents bacterial infection after sinus floor augmentation. (30) Bolukbasi et al.(31) reported that the effectiveness of PRF is mainly based on the characteristics of the graft combined with PRF and it is effective in the early phases of the bone regeneration process. There is yet no consensus on the terms and algorithm of the use of the combination of PRF with other grafting materials. However, several authors reported that new bone regeneration was observed after sinus floor augmentation with the combination of PRF and bovine bone graft or demineralized bone allograft. $(17,18,31)$ It is suggested that PRF should be combined with additional bone grafting materials if the bucco-palatal dimension of the sinus exceeds $15 \mathrm{~mm}$.(32) In the current study, all included cases had increased bucco-palatal dimensions due to extensive pneumatization and atrophy in the posterior maxilla.

\section{Conclusion}

The use of the combination of PRF and demineralized cortico-cancellous bone allograft may be a working option in the sinus floor augmentation with simultaneous dental implant placement. The success of dental implants and the bone graft is related to the good primer stability in the severely atrophic posterior maxilla. The primer stability of simultaneously placed dental implants may be secured by using a wider dental implant than the osteotomy width.

\section{References}

1. Del Fabbro M, Testori T, Francetti L, Weinstein R. Systematic review of survival rates for implants placed in the grafted maxillary sinus. The International journal of periodontics \& restorative dentistry. 2004;24(6):565-77.

2. Klijn RJ, Meijer GJ, Bronkhorst EM, Jansen JA. A meta-analysis of histomorphometric results and graft healing time of various biomaterials compared to autologous bone used as sinus floor augmentation material in humans. Tissue engineering Part B, Reviews. 2010;16(5):493-507.

3. Kolerman R, Nissan J, Rahmanov M, Vered H, Cohen O, Tal H. Comparison between mineralized cancellous bone allograft and an alloplast material for sinus augmentation: A split mouth histomorphometric study. Clinical implant dentistry and related research. 2017;19(5):812-20.

4. Guerrero JS, Al-Jandan BA. Allograft for maxillary sinus floor augmentation: a retrospective study of 90 cases. Implant dentistry. 2012;21(2):136-40.

5. Valentini P, Abensur D. Maxillary sinus floor elevation for implant placement with demineralized freeze- 
dried bone and bovine bone (Bio-Oss): a clinical study of 20 patients. The International journal of periodontics \& restorative dentistry. 1997;17(3):232-41.

6. Browaeys H, Bouvry P, De Bruyn H. A literature review on biomaterials in sinus augmentation procedures. Clinical implant dentistry and related research. 2007;9(3):166-77.

7. Boëck-Neto RJ, Gabrielli M, Lia R, Marcantonio E, Shibli JA, Marcantonio E, Jr. Histomorphometrical analysis of bone formed after maxillary sinus floor augmentation by grafting with a combination of autogenous bone and demineralized freeze-dried bone allograft or hydroxyapatite. Journal of periodontology. 2002;73(3):266-70.

8. Landi L, Pretel RW, Jr., Hakimi NM, Setayesh R. Maxillary sinus floor elevation using a combination of DFDBA and bovine-derived porous hydroxyapatite: a preliminary histologic and histomorphometric report. The International journal of periodontics \& restorative dentistry. 2000;20(6):574-83.

9. Tilaveridis I, Lazaridou M, Zouloumis L, Dimitrakopoulos I, Tilaveridis V, Tilaveridou S. The use of mineralized bone allograft as a single grafting material in maxillary sinus lifting with severely atrophied alveolar ridge $(1-3 \mathrm{~mm})$ and immediately inserted dental implants. A 3- up to 8-year retrospective study. Oral and maxillofacial surgery. 2018;22(3):267-73.

10. Chaushu G, Mardinger O, Calderon S, Moses O, Nissan J. The use of cancellous block allograft for sinus floor augmentation with simultaneous implant placement in the posterior atrophic maxilla. Journal of periodontology. 2009;80(3):422-8.

11. Peleg M, Mazor Z, Chaushu G, Garg AK. Sinus floor augmentation with simultaneous implant placement in the severely atrophic maxilla. Journal of periodontology. 1998;69(12):1397-403.

12. Ulm CW, Solar P, Gsellmann B, Matejka M, Watzek G. The edentulous maxillary alveolar process in the region of the maxillary sinus-a study of physical dimension. International journal of oral and maxillofacial surgery. 1995;24(4):279-82.

13. Liu R, Yan M, Chen S, Huang W, Wu D, Chen J. Effectiveness of Platelet-Rich Fibrin as an Adjunctive Material to Bone Graft in Maxillary Sinus Augmentation: A Meta-Analysis of Randomized Controlled Trails. BioMed research international. 2019;2019:7267062.

14. Castro AB, Cortellini S, Temmerman A, Li X, Pinto N, Teughels W, et al. Characterization of the Leukocyte- and Platelet-Rich Fibrin Block: Release of Growth Factors, Cellular Content, and Structure. The International journal of oral \& maxillofacial implants. 2019;34(4):855-64.

15. Dohan DM, Choukroun J, Diss A, Dohan SL, Dohan AJ, Mouhyi J, et al. Platelet-rich fibrin (PRF): a second-generation platelet concentrate. Part II: platelet-related biologic features. Oral surgery, oral medicine, oral pathology, oral radiology, and endodontics. 2006;101(3):e45-50.

16. Dohan DM, Choukroun J, Diss A, Dohan SL, Dohan AJ, Mouhyi J, et al. Platelet-rich fibrin (PRF): a second-generation platelet concentrate. Part III: leucocyte activation: a new feature for platelet concentrates? Oral surgery, oral medicine, oral pathology, oral radiology, and endodontics. 2006;101(3):e51-5.

17. Peker E, Karaca IR, Yildirim B. Experimental Evaluation of the Effectiveness of Demineralized Bone Matrix and Collagenated Heterologous Bone Grafts Used Alone or in Combination with Platelet-Rich Fibrin on Bone Healing in Sinus Floor Augmentation. The International journal of oral \& maxillofacial implants. 2016;31(2):e24-31.

18. Pichotano EC, de Molon RS, de Souza RV, Austin RS, Marcantonio E, Zandim-Barcelos DL. Evaluation of L-PRF combined with deproteinized bovine bone mineral for early implant placement after maxillary sinus augmentation: A randomized clinical trial. Clinical implant dentistry and related research. 2019;21(2):253-62.

19. Lundgren S, Cricchio G, Hallman M, Jungner M, Rasmusson L, Sennerby L. Sinus floor elevation procedures to enable implant placement and integration: techniques, biological aspects and clinical outcomes. 
Periodontology 2000. 2017;73(1):103-20.

20. Mardinger O, Nissan J, Chaushu G. Sinus floor augmentation with simultaneous implant placement in the severely atrophic maxilla: technical problems and complications. Journal of periodontology. 2007;78(10):18727.

21. Diserens V, Mericske E, Mericske-Stern R. Radiographic analysis of the transcrestal sinus floor elevation: short-term observations. Clinical implant dentistry and related research. 2005;7(2):70-8.

22. Mazzocco F, Lops D, Gobbato L, Lolato A, Romeo E, del Fabbro M. Three-dimensional volume change of grafted bone in the maxillary sinus. The International journal of oral \& maxillofacial implants. 2014;29(1):17884.

23. Klein GG, Curvello VP, Dutra RA, Simeão SP, Santos PL, Gulinelli JL, et al. Bone Volume Changes After Sinus Floor Augmentation with Heterogenous Graft. The International journal of oral \& maxillofacial implants. 2016;31(3):665-71.

24. Kiliç SC, Güngörmüs M. Cone Beam Computed Tomography Assessment of Maxillary Sinus Floor Augmentation Using Beta-Tricalcium Phosphate Alone or in Combination with Platelet-Rich Plasma: A Randomized Clinical Trial. The International journal of oral \& maxillofacial implants. 2016;31(6):1367-75.

25. Gultekin BA, Cansiz E, Borahan O, Mangano C, Kolerman R, Mijiritsky E, et al. Evaluation of Volumetric Changes of Augmented Maxillary Sinus With Different Bone Grafting Biomaterials. The Journal of craniofacial surgery. 2016;27(2):e144-8.

26. Ohe JY, Kim GT, Lee JW, Al Nawas B, Jung J, Kwon YD. Volume stability of hydroxyapatite and $\beta$-tricalcium phosphate biphasic bone graft material in maxillary sinus floor elevation: a radiographic study using 3D cone beam computed tomography. Clinical oral implants research. 2016;27(3):348-53.

27. Cosso MG, de Brito RB, Jr., Piattelli A, Shibli JA, Zenóbio EG. Volumetric dimensional changes of autogenous bone and the mixture of hydroxyapatite and autogenous bone graft in humans maxillary sinus augmentation. A multislice tomographic study. Clinical oral implants research. 2014;25(11):1251-6.

28. Gultekin BA, Borahan O, Sirali A, Karabuda ZC, Mijiritsky E. Three-Dimensional Assessment of Volumetric Changes in Sinuses Augmented with Two Different Bone Substitutes. BioMed research international. 2016;2016:4085079.

29. Broder J, Preston R. Chapter 1 - Imaging the Head and Brain. In: Broder J, editor. Diagnostic Imaging for the Emergency Physician. Saint Louis: W.B. Saunders; 2011. p. 1-45.

30. Everts PA, Overdevest EP, Jakimowicz JJ, Oosterbos CJ, Schönberger JP, Knape JT, et al. The use of autologous platelet-leukocyte gels to enhance the healing process in surgery, a review. Surgical endoscopy. 2007;21(11):2063-8.

31. Bolukbasi N, Ersanlı S, Keklikoglu N, Basegmez C, Ozdemir T. Sinus Augmentation With Platelet-Rich Fibrin in Combination With Bovine Bone Graft Versus Bovine Bone Graft in Combination With Collagen Membrane. The Journal of oral implantology. 2015;41(5):586-95.

32. Miron RJ, Pikos MA. Sinus Augmentation Using Platelet-Rich Fibrin With or Without a Bone Graft: What Is the Consensus? Compendium of continuing education in dentistry (Jamesburg, NJ : 1995). 2018;39(6):355-61; quiz 62 .

\section{Figure Legends}

Figure 1. PRF was cut into small fragments and mixed with particulate demineralized cortico-cancellous bone allograft in a sterile container immediately before usage.

Figure 2. A- The marking of the cavity borders was commenced on the anterior part of the sinus cavity with a pink border marking tool. B- The whole sinus cavity was marked with the marking tool. C- The 
sinus cavity in the remaining consecutive tomographic sections were marked with the pink marking tool. DThe volume of the cavity was determined by a green volume measurement tool after all borders were marked in the axial section.

Figure 3. The deficient areas in the sagittal sections were observed and corrected by the same marking and measurement tool.

Figure 4. The deficient areas in the coronal sections were observed and corrected by the same marking and measurement tool.

Figure 5. The final volume of the sinus cavity was determined with the reconstruction feature after all corrections were made in sagittal and coronal sections.

Figure 6. The residual ridge height was measured on the mid-crestal region of mid-sagittal (A) and mid-coronal (B) planes with a measurement tool that automatically shows the diameter in $\mathrm{mm}$. The postoperative ridge height was measured on mid-sagittal (C) and mid-coronal (D) planes in close proximity to dental implants placed simultaneously with sinus floor augmentation.

Figure 7. Residual and post-operative ridge heights of all study patients.

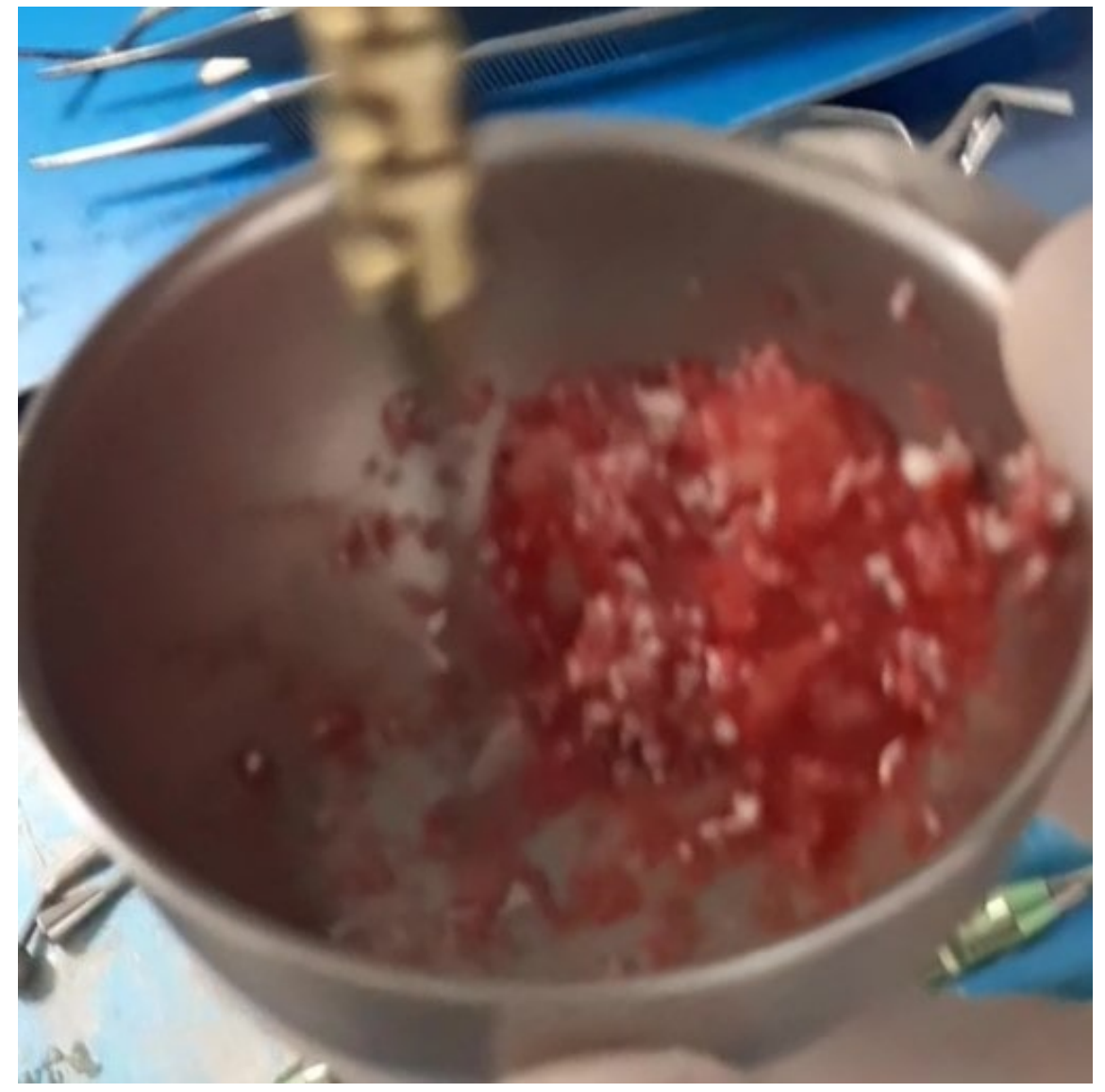



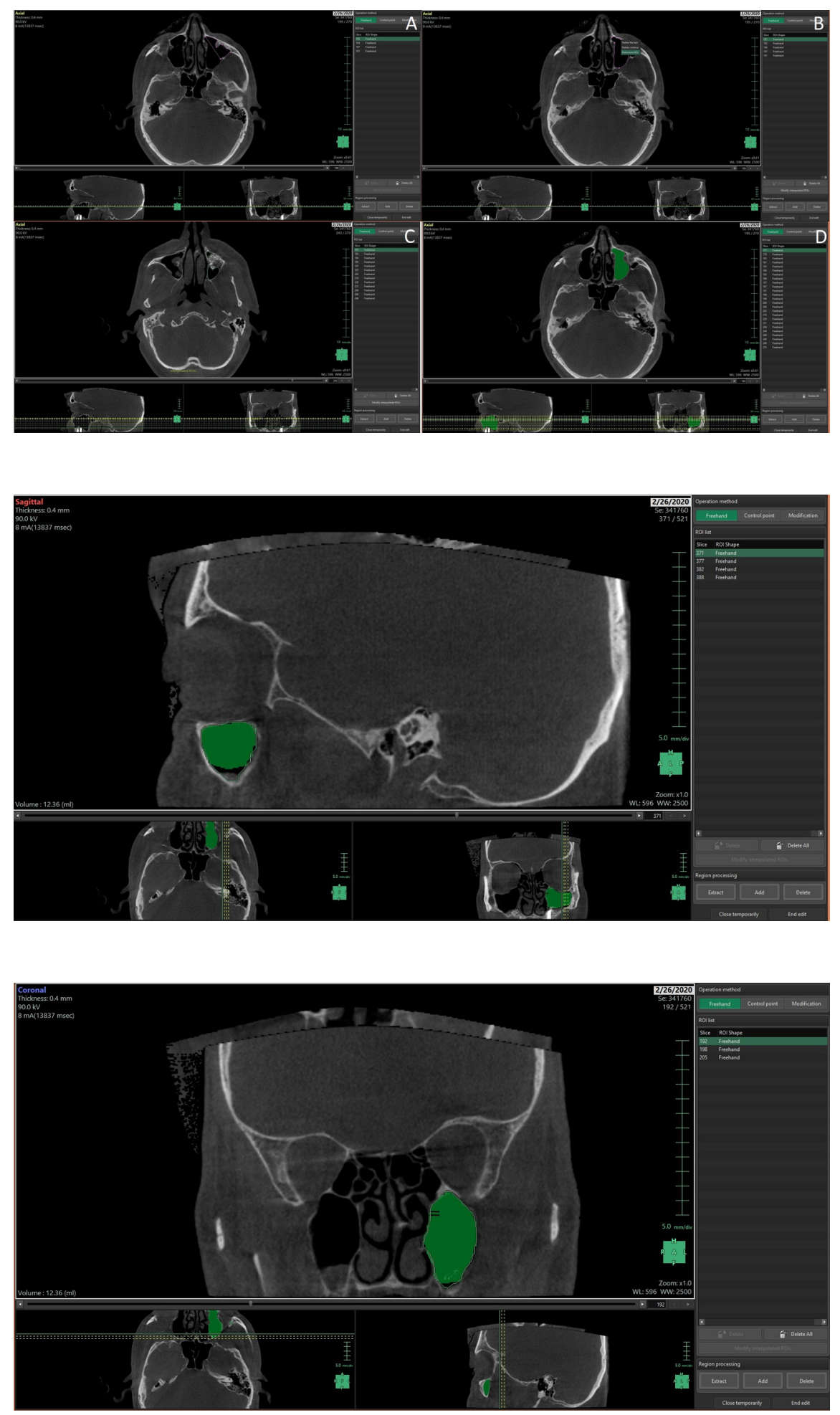

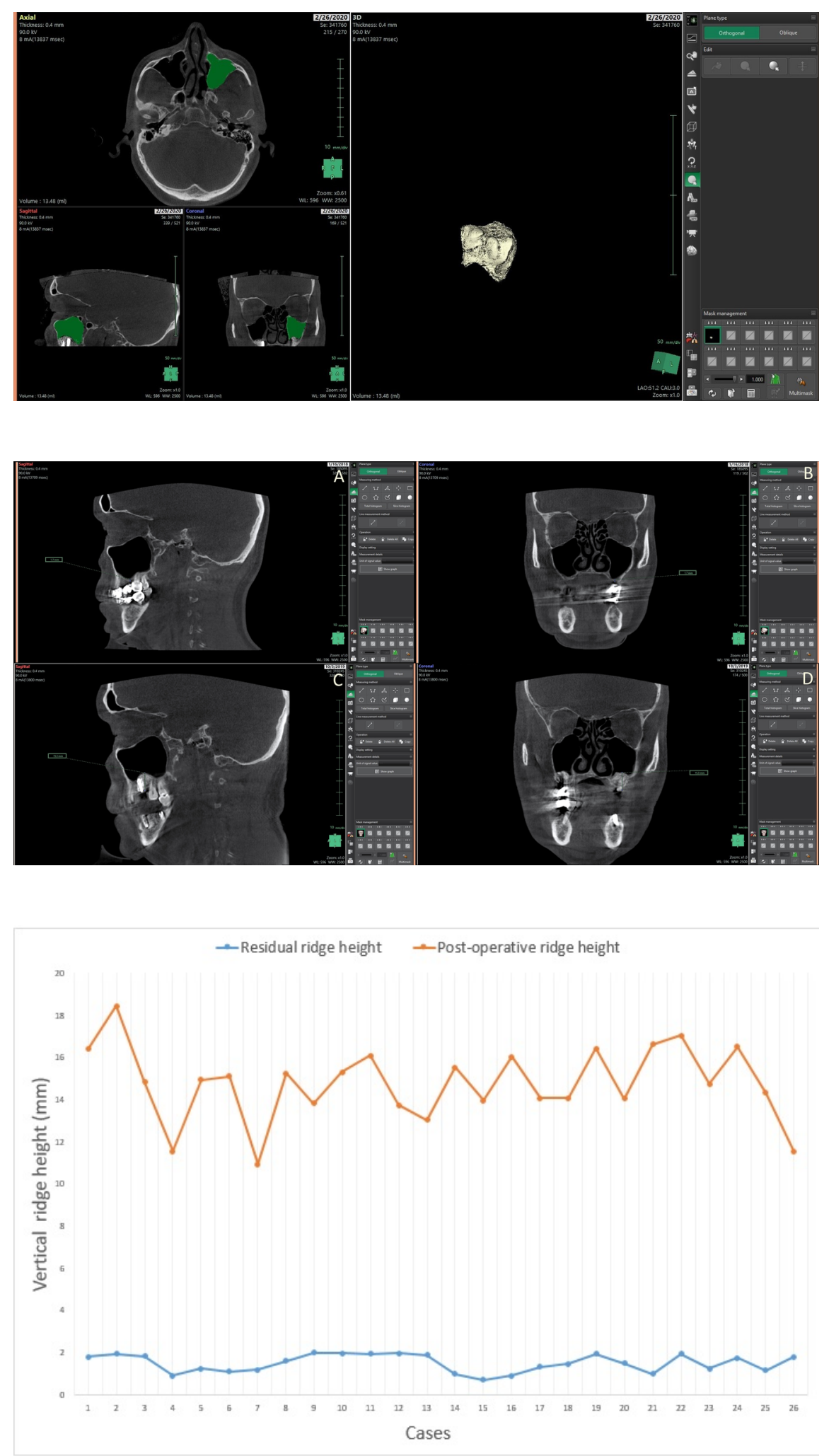

Hosted file 
Table 1.pdf available at https://authorea.com/users/388728/articles/503490-the-volumetricevaluation-of-sin\%C3\%BCs-floor-augmentation-using-platelet-rich-fibrin-and-allogenicbone-graft-mixture-with-simultaneous-dental-implant-placement-in-severely-atrophicposterior-maxilla

\section{Hosted file}

Table 2.pdf available at https://authorea.com/users/388728/articles/503490-the-volumetricevaluation-of-sin\%C3\%BCs-floor-augmentation-using-platelet-rich-fibrin-and-allogenicbone-graft-mixture-with-simultaneous-dental-implant-placement-in-severely-atrophicposterior-maxilla

\section{Hosted file}

Table 3.pdf available at https://authorea.com/users/388728/articles/503490-the-volumetricevaluation-of-sin\%C3\%BCs-floor-augmentation-using-platelet-rich-fibrin-and-allogenicbone-graft-mixture-with-simultaneous-dental-implant-placement-in-severely-atrophicposterior-maxilla

\section{Hosted file}

Table 4.pdf available at https://authorea.com/users/388728/articles/503490-the-volumetricevaluation-of-sin\%C3\%BCs-floor-augmentation-using-platelet-rich-fibrin-and-allogenicbone-graft-mixture-with-simultaneous-dental-implant-placement-in-severely-atrophicposterior-maxilla 